\title{
Risk factors for admission to hospital with laboratory-confirmed influenza in young children: birth cohort study
}

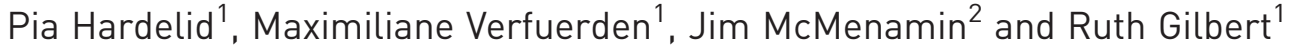 \\ Affiliations: ${ }^{1}$ Population, Policy and Practice Programme, UCL Great Ormond Street Institute of Child Health, \\ London, UK. ${ }^{2}$ Health Protection Scotland, Glasgow, UK. \\ Correspondence: Pia Hardelid, Population, Policy and Practice Programme, UCL Great Ormond Street \\ Institute of Child Health, 30 Guilford Street, London WC1N 1EH, UK. E-mail: p.hardelidaucl.ac.uk
}

@ERSpublications

Older siblings pose risk of serious influenza for babies and toddlers http://ow.ly/RQDS30dbtIo

Cite this article as: Hardelid P, Verfuerden M, McMenamin J, et al. Risk factors for admission to hospital with laboratory-confirmed influenza in young children: birth cohort study. Eur Respir J 2017; 50: 1700489 [https://doi.org/10.1183/13993003.00489-2017].

ABSTRACT We determined risk factors for influenza hospital admission in children aged $<2$ years to guide the design of paediatric vaccination programmes.

We linked all singleton live births in Scotland from 2007 to 2015 to hospital administrative data and influenza laboratory reports. Cox proportional hazard models were used to identify birth and family risk factors for influenza admissions.

There were 1115 influenza admissions among 424048 children. 85.1\% of admitted children were born at term and were not in a high-risk group. Presence of an older sibling was strongly associated with increased risk of influenza admission, particularly for children aged $<6$ months: hazard ratio for second- versus firstborn child was 2.02 (95\% CI 1.52-2.69). Maternal age <30 years and birth during autumn (age $<6$ months) or spring (age 6-23 months) were also associated with admission risk.

Targeting vaccination programmes to high-risk children will not prevent the vast majority of influenza admissions. Parents of children aged $<2$ years should be advised that vaccination of older siblings will protect younger children against influenza infection. As evidence of the impact of the universal influenza vaccine programme emerges, there may be a need to reconsider universal influenza vaccination in children aged 6 months to 2 years in the UK.

This article has supplementary material available from erj.ersjournals.com

Received: March 092017 | Accepted after revision: June 222017

Support statement: This study was funded by a National Institute for Health Research Postdoctoral Fellowship to P. Hardelid, reference number PDF-2013-06-004. The views expressed in this publication are those of the author(s) and not necessarily those of the NHS, the National Institute for Health Research or the Department of Health. The study sponsor had no role in the study design, data analysis, interpretation, writing the report, or in the decision to submit the paper for publication. The corresponding author had full access to all the data in the study and had final responsibility for the decision to submit for publication. Funding information for this article has been deposited with the Crossref Funder Registry.

Conflict of interest: Disclosures can be found alongside this article at erj.ersjournals.com

Copyright @ERS 2017 


\section{Introduction}

Between $3 \%$ and $11 \%$ of children aged $<2$ years in developed countries acquire influenza-associated illness every year [1], creating a major burden on both primary and secondary care services [2]. This has led many countries to implement influenza immunisation programmes. Inactivated influenza vaccines are licensed for use in children aged $\geqslant 6$ months, and live attenuated vaccines for children aged $\geqslant 2$ years. Children aged $<6$ months are at highest risk of influenza hospital admission [3]; however, no influenza vaccines are licensed for this age group due to reports of limited effectiveness [4]. Instead, the preferred strategy for preventing influenza in this age group is through the vaccination of pregnant women. Although clinical trials and observational studies have both reported a protective effect of maternal influenza vaccination on infections in babies aged $<6$ months [5-7], this appears to be limited to the first 8 weeks of life [8].

Paediatric influenza vaccination policies vary between countries (table 1). The United States and Canada use a universal approach, while the majority of European countries employ a targeted strategy aimed at reducing the burden of influenza morbidity among children at high risk of complications due to underlying chronic conditions.

The evidence regarding whether universal influenza vaccination programmes lead to higher uptake in high-risk groups is conflicting $[15,16]$; however, both universal and targeted programmes have lower rates of uptake than other routine childhood vaccination programmes [17-19]. In the UK, $18.6 \%$ of children aged between 6 months and 2 years in a high-risk group were vaccinated [20], whereas 32.9-57.1\% of preschool children were vaccinated under the universal programme in 2015-2016, depending on UK country [21]. Likewise, vaccination uptake in pregnant women in the UK and many other countries remains $<50 \%[20,22,23]$. Improved evidence is needed to guide policy about which children should be offered influenza vaccination and to improve uptake in those children most likely to benefit.

A recent systematic review of observational studies found that children aged $<2$ years, born prematurely, or with neurological conditions, immunosuppression or diabetes were at significantly increased risk of

\section{TABLE 1 Influenza vaccination policy in children in selected developed countries

$\begin{array}{ccc}\text { Country [reference] } & \begin{array}{c}\text { Influenza vaccination } \\ \text { recommendation for } \\ \text { children }\end{array} & \text { Description }\end{array}$

USA [9], Canada [10]

Australia [11, 12]

UK

Finland [13]

Slovakia [13]

Germany, France, Italy, Spain, Sweden, Denmark [13] and New Zealand [14]
Mixed

Mixed

Universal

Universal

Mixed

Targeted
Age $\geqslant 6$ months: vaccination recommended for all children, but priority for vaccine supply given to children aged 6-59 months and children in high-risk groups and (in Canada) Aboriginal children

Age $\geqslant 6$ months: vaccination recommended for all children, but offered free to Aboriginal/Torres Strait Islander children aged 6-59 months and children in high-risk groups

Children in Western Australia aged 659 months are offered free vaccination Age 6-23 months: targeted vaccination offered to children in high-risk groups Age 24 months -16 years: universal offer of vaccination (since September 2013)

Age 6-35 months: universal offer of vaccination to children

Age $\geqslant 36$ months: targeted vaccination offered to children in high-risk groups Age 6 months -12 years: universal offer of vaccination to children

Age $\geqslant 12$ years: targeted vaccination offered to children in high-risk groups Age $\geqslant 6$ months: targeted vaccination for children in high-risk groups 
influenza hospital admission [24]. There are few studies regarding risk factors not associated with underlying chronic conditions; however, young maternal age ( $<26$ years), poverty/education level, minority ethnic group, smoking in the home and number of children in the household have been identified as risk factors for influenza hospital admission in three case-control studies [25-27]. There are no studies in children aged $<6$ months.

We performed a cohort study to determine risk factors for influenza hospital admissions in children aged $<2$ years throughout Scotland. We focussed on examining birth and family risk factors that are readily identifiable and could be included in programmes that aim to target the offer of vaccination and/or to improve the uptake of vaccination among children and pregnant women.

\section{Methods}

Data sources, study period and population

We analysed a national cohort of all singleton births in Scotland between October 2007 and April 2015 by linking electronic birth and death registration data held by National Records for Scotland to hospital admission records (Scottish Morbidity Records (SMR-01)), Scottish Birth Records, Scottish Maternity Records (SMR-02) and the national laboratory surveillance database, Electronic Communication of Surveillance in Scotland (ECOSS). ECOSS contains details of all positive detections reported to Health Protection Scotland (HPS), the national public health agency, from microbiology laboratories serving primary and secondary care in the National Health Service (NHS). Deterministic linkage between databases was performed by the Electronic Data Research and Innovation Service (eDRIS) using the Community Health Index number, a unique individual identifier assigned at birth and recorded on all interactions with the Scottish NHS, including on ECOSS. This study was approved by the Public Benefit and Privacy Panel for Health and Social Care (reference number 1516-0405).

Children were followed from birth or the start of the follow-up period (whichever occurred last) until 2 years of age, date of death or outmigration, or the end of the follow-up period, whichever occurred first. We excluded births before 24 weeks of gestation to minimise misclassification of stillbirths, and births to nonresident Scottish mothers. We measured influenza-confirmed admissions during the follow-up period from September 1, 2009 to May 31, 2015. Laboratory surveillance for influenza through ECOSS became routine during the summer of 2009, and 2014/2015 was the last full influenza season in the dataset.

We present results separately for infants aged $<6$ months and children aged 6-23 months. For both age groups, we assumed that children had not been vaccinated. While true for infants aged $<6$ months, we estimate that $0.6 \%$ of children aged 6-23 months were misclassified as unvaccinated under this assumption. Vaccine coverage data from primary care indicate that $3 \%$ of 2 -year-olds would be in a clinical risk group and $18.5 \%$ of them would be vaccinated [20].

\section{Outcome: influenza-confirmed hospital admission}

We defined a laboratory-confirmed influenza admission as an emergency hospital admission in SMR-01 with a linked influenza-positive ECOSS episode, where the specimen date was $\leqslant 7$ days before or after the admission date, and the primary diagnosis was not an injury (International Classification of Diseases version 10 (ICD-10) codes S00-T79). We allowed any sample that was positive for influenza (irrespective of place of collection, type of sample or testing method) between August 25, 2009 and June 7, 2015 that met these criteria to be linked to an emergency admission to allow for tests performed up to a week before or after an admission, and to include both seasonal and pandemic strains of influenza. A patient may test positive for the same pathogen multiple times during their illness. Therefore, HPS derives infection episodes to identify positive laboratory tests relating to the same illness period. If more than one emergency hospital admission linked to an ECOSS episode within the 14-day period, the admission with the admission date closest to the ECOSS specimen date was selected as the influenza-confirmed admission.

\section{Risk factors}

We examined risk factors that are routinely recorded in infant delivery and hospitalisation records and are known to be associated with morbidity from respiratory tract infections. Gestational age was coded into a three-category variable to ensure sufficient numbers for analysis: preterm ( $<37$ weeks), term (37-40 weeks) and post-term $(\geqslant 41$ weeks). Season of birth was coded into quarters of the year (January-March, AprilJune, July-September and October-December). We examined presence of older siblings (coded using parity information into none, one or two or more), maternal age (grouped as <20, 20-29, 30-39 and $\geqslant 40$ years) and quintiles of the Scottish Index of Multiple Deprivation, which is based on area-level indicators of deprivation for areas of 500-1000 people [28].

We adjusted all models for presence of high-risk conditions. High-risk conditions were identified using ICD-10 diagnostic codes recorded in either the Scottish Birth Record (neonatal diagnoses) or in SMR-01 
until 6 months of age. The code list for identifying high-risk conditions builds on a previously published list [29], which we extended by cross-checking against a list of conditions used to define high-risk groups in primary care [30], and including chromosomal abnormalities (online supplementary table S1). Since not all high-risk conditions are recorded in hospital databases, we further adjusted for prolonged postnatal stay in hospital (defined as $>14$ days) as an indicator of birth trauma or neonatal intensive care with potential neurological and/or respiratory sequelae.

\section{Statistical analyses}

We included only the first influenza-confirmed admission for each child within each of the two age groups. We used Stata (version 13; StataCorp, College Station, TX, USA) for statistical analyses. We calculated influenza-confirmed admission rates per 1000 child-years by age group ( $<6$ months and 6-23 months), by each exposure variable and by influenza season (defined as September 1 in year $x$ to May 31 in year $x+1$ ). In addition, the dominant strains in each season were derived from ECOSS [31].

We used Cox proportional hazards regression models to examine the association between the exposure variables and the risk of influenza-confirmed hospital admission. An admitted child was censored at their admission date. The proportional hazards assumption was checked using cumulative hazard plots. All exposure variables were included in the models a priori, and associations with a Wald test $\mathrm{p}<0.05$ were considered to be statistically significant. Due to a non-negligible proportion of children with missing data on risk factors, we used multiple imputation with 15 imputations [32]. The main results are based on the imputed datasets. We performed a sensitivity analysis where we additionally classified children who had a clinically coded influenza admission without a linked ECOSS-positive episode as having had an influenza-confirmed admission. Clinically coded influenza admissions were identified using ICD-10 codes J09-J11 in any of the diagnostic fields.

We estimated population-attributable fractions (PAFs) for all risk factors that were significantly associated with the outcome and considered amenable via vaccination policy or health/social policy: maternal age, number of older siblings and season of birth, and in addition for 6-23-month-olds, the presence of high-risk conditions, smoking during pregnancy and gestational age. PAFs were estimated using the punafcc function with robust standard errors [33], using the complete case models.

\section{Results}

This study included a birth cohort of 424048 children born in Scotland and 636428 child-years of follow-up. There were 1115 influenza-confirmed admissions during the follow-up period in children aged $<2$ years. 1109 children had one influenza-confirmed admission during the study period; three children had two. The linkage between hospital admission and laboratory data is summarised in figure 1. Out of the 849 influenza-positive ECOSS samples that did not link to an admission within the birth cohort, 174 (20.5\%) were collected in general practice surgeries and 675 (79.5\%) were collected in hospital (emergency or outpatients department) or elsewhere (online supplementary figure S1). These unlinked samples were therefore unlikely to relate to a hospital admission. Online supplementary table S2 shows the characteristics of the cohort children, including the proportion with missing data. $<3 \%$ of children in the birth cohort aged 6-23 months were in a clinical risk group and would therefore qualify for influenza vaccination (online supplementary table S2).

Of the 1115 confirmed influenza admissions, 414 (37.1\%) had a hospital diagnosis indicating influenza (online supplementary figure S2) and 344 (30.9\%) had a primary diagnosis which was not from the respiratory conditions chapter of the ICD-10 (online supplementary figure S3).

Admission rates for confirmed influenza during influenza seasons (per 1000 child-years) were 2.68 (95\% CI 2.40-2.99) in children aged $<6$ months and 2.09 (1.95-2.49) in children aged 6-23 months. Influenza-confirmed admission rates peaked in the 2012/2013 influenza season in both age groups (figure 2). Admission rates using diagnosis codes to define influenza admissions were significantly lower at 1.89 (1.65-2.14) and 1.09 (0.99-1.20) for children aged <6 months and 6-23 months, respectively. Rates of influenza-confirmed admissions peaked in January (online supplementary figure S4).

Crude admission rates were significantly higher among children in high-risk groups (table 2); however, they represented a small minority of admitted children: 895 (85.1\%) of the 1052 children who were admitted with at least one influenza-confirmed admission and had their gestational age recorded were born at or after term without recorded high-risk conditions. In fully adjusted models for children aged $<6$ months, born between July and September, having at least one older sibling and maternal age $<30$ years were most strongly associated with an increased risk of influenza-confirmed admission (table 3).

Among children aged 6-23 months, premature birth, season of birth (January-June), high-risk conditions, maternal smoking during pregnancy, presence of one or more older siblings, maternal age <30 years 

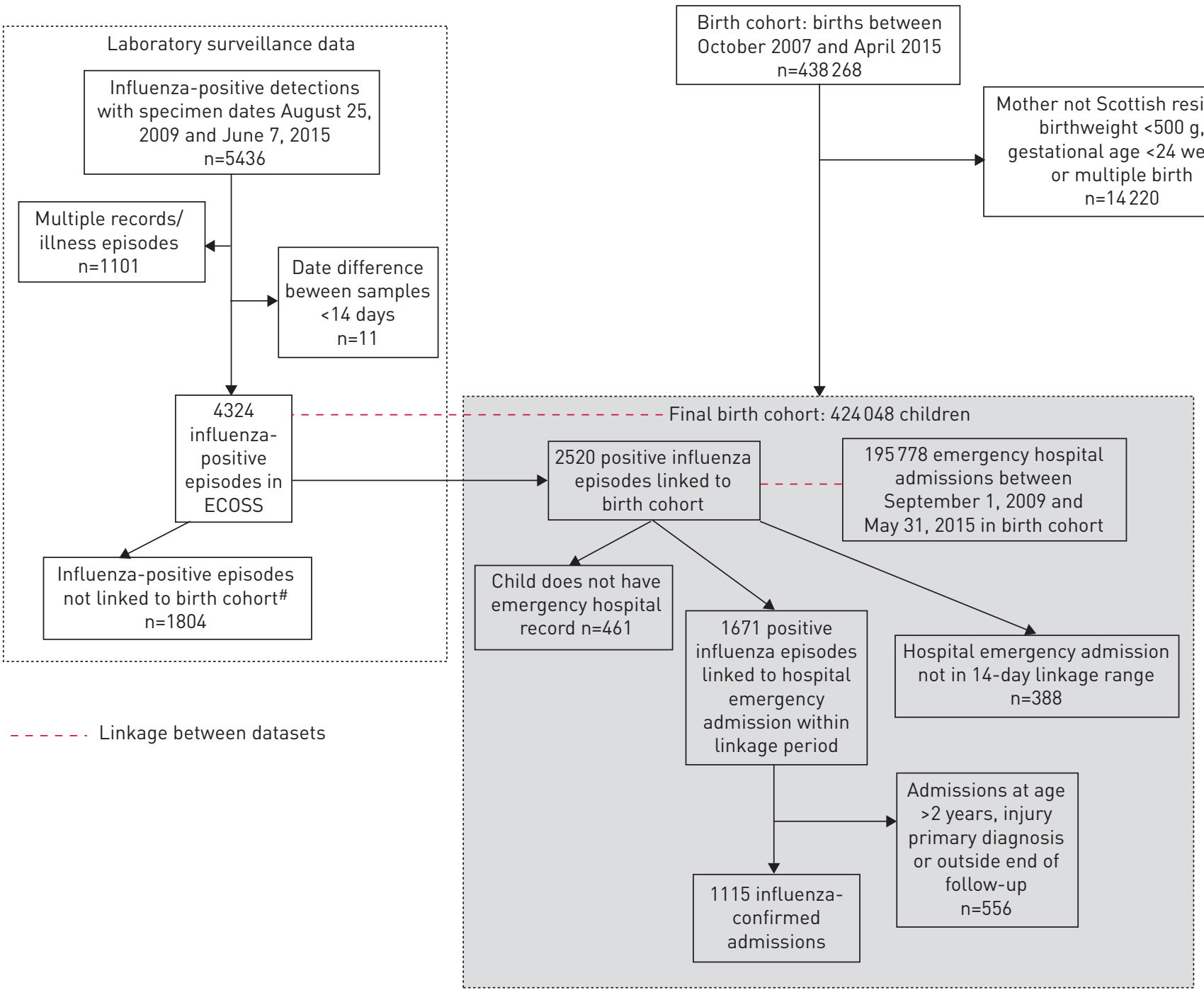

FIGURE 1 Flowchart of linkage outcomes between birth cohort, emergency hospital admissions and laboratory-confirmed influenza-positive episodes. " : 1699 (94.2\%) of these samples were from children who were born before the earliest date of birth in the cohort.

and having a prolonged postnatal hospital stay were associated with a significantly increased risk of influenza-confirmed admission (table 4). The hazard ratios for presence of siblings, season of birth and maternal age group were weaker for 6-23-month-olds than for children aged $<6$ months.

These results remained very similar in the complete case analyses (online supplementary table S3). For children aged $<6$ months old, the hazard ratio for prematurity (born at $<37$ weeks) became statistically significant when we classified admissions with a clinical code indicating influenza as an influenza-confirmed admission (online supplementary table S4).

The highest proportion of cases could be prevented by reducing variation in risk according to season of birth, number of siblings and maternal age in both age groups (table 5). Targeted strategies towards high-risk groups would only prevent $4-6 \%$ of cases.

\section{Discussion}

Presence of an older sibling, season of birth, maternal age $<30$ years and presence of a high-risk condition were all risk factors for laboratory-confirmed influenza hospital admission in children aged $<2$ years. Preterm birth was a significant risk factor for influenza admission among children aged 6-23 months. However, the low prevalence of high-risk conditions and preterm births resulted in low estimated population-attributable risk fractions for these characteristics. Targeting vaccination at children with these risk factors would be expected to achieve a very small reduction in the number of influenza admissions among all children in the age group. Among children aged $<6$ months, presence of an older sibling 


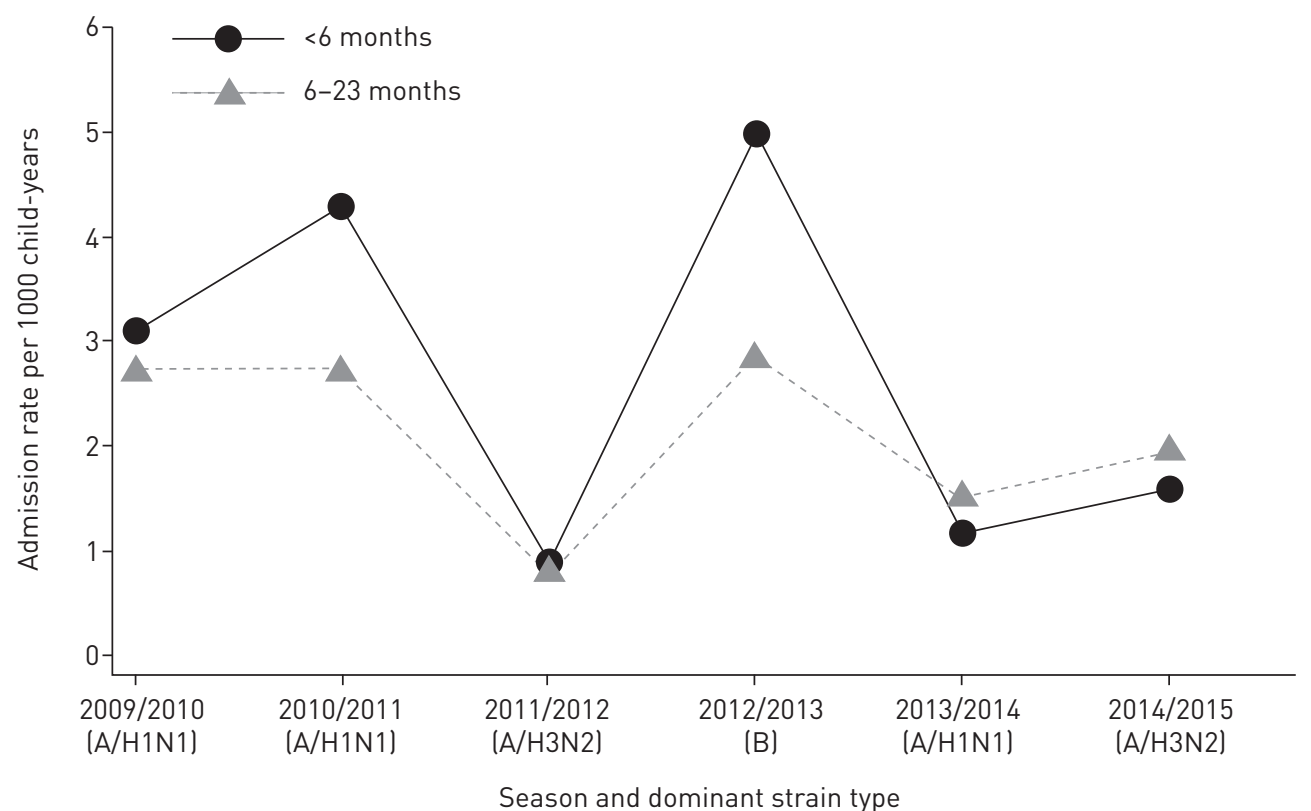

FIGURE 2 Influenza-confirmed admission rates (per 1000 child-years) by influenza season (with dominant strain typel and age group.

and birth during autumn accounted for the highest population-attributable risk. For children aged 6-23 months, the population-attributable risk was relatively low $(<20 \%)$ for each risk factor.

We used a national birth cohort of $>400000$ children constructed using linked administrative health databases, and exploited linkage between hospital and laboratory surveillance databases to define influenza-confirmed hospital admissions. This avoided reliance on diagnostic coding, which has been shown to have low sensitivity in previous studies $[34,35]$ and would have underestimated admission rates in our study by up to $50 \%$. The large sample size and national coverage allowed us to examine key risk factors that could be readily targeted and monitored in a vaccination programme.

There is no national testing protocol in the UK for children presenting to hospital with symptoms of respiratory infections. Our ECOSS database extract included only influenza-positive test results, hence we could not examine how testing probabilities vary by child characteristics. The likelihood of sample collection is likely to increase with severity of illness, and we may have misclassified children who were admitted with influenza but did not have a diagnostic sample taken. In addition, testing may be more likely among children with high-risk conditions or who were born prematurely. If this is the case, it would mean that the true population-attributable risk for high-risk conditions may be even lower than our estimate. Since our method is likely to underestimate the true number of children admitted to hospital with influenza, we performed sensitivity analysis including children with a clinical code mentioning influenza (but no recorded positive test result), which yielded similar results. In the absence of universal sampling of all children presenting to hospital with respiratory symptoms or fever, the methods used here are likely to yield the most comprehensive and unbiased picture of risk factors for influenza hospitalisations in young children achievable using routinely collected administrative health data.

A second limitation is that we could not take into account vaccination status. During the study period there was no individual national data collection on paediatric influenza immunisation in any UK country, although recording is improving over time in Scotland. However, only a small minority of eligible 623-month-old children are vaccinated. Such misclassification would be expected to bias hazard ratios for clinical risk factors towards the null effect. Further, although we had information on parity, we could not examine the effect of sibling ages. Childcare attendance is strongly associated with an increased risk of respiratory tract infections in early childhood [36]; however, there is no national register of preschool childcare in Scotland. Finally, we could only identify children in high-risk groups based on hospital records. This may miss children whose chronic conditions are managed in primary care, such as children with severe wheeze. However, we also adjusted for prolonged postnatal stay for allow potential underascertainment of children in risk groups.

The rates of hospital admission during influenza seasons reported in our study are similar to previous studies based on laboratory confirmed influenza in the US and England [35, 37]. In addition, our 
TABLE 2 Number of influenza-confirmed admissions and rates by birth and family characteristics in each of the two study cohorts, September 2009 to May 2015

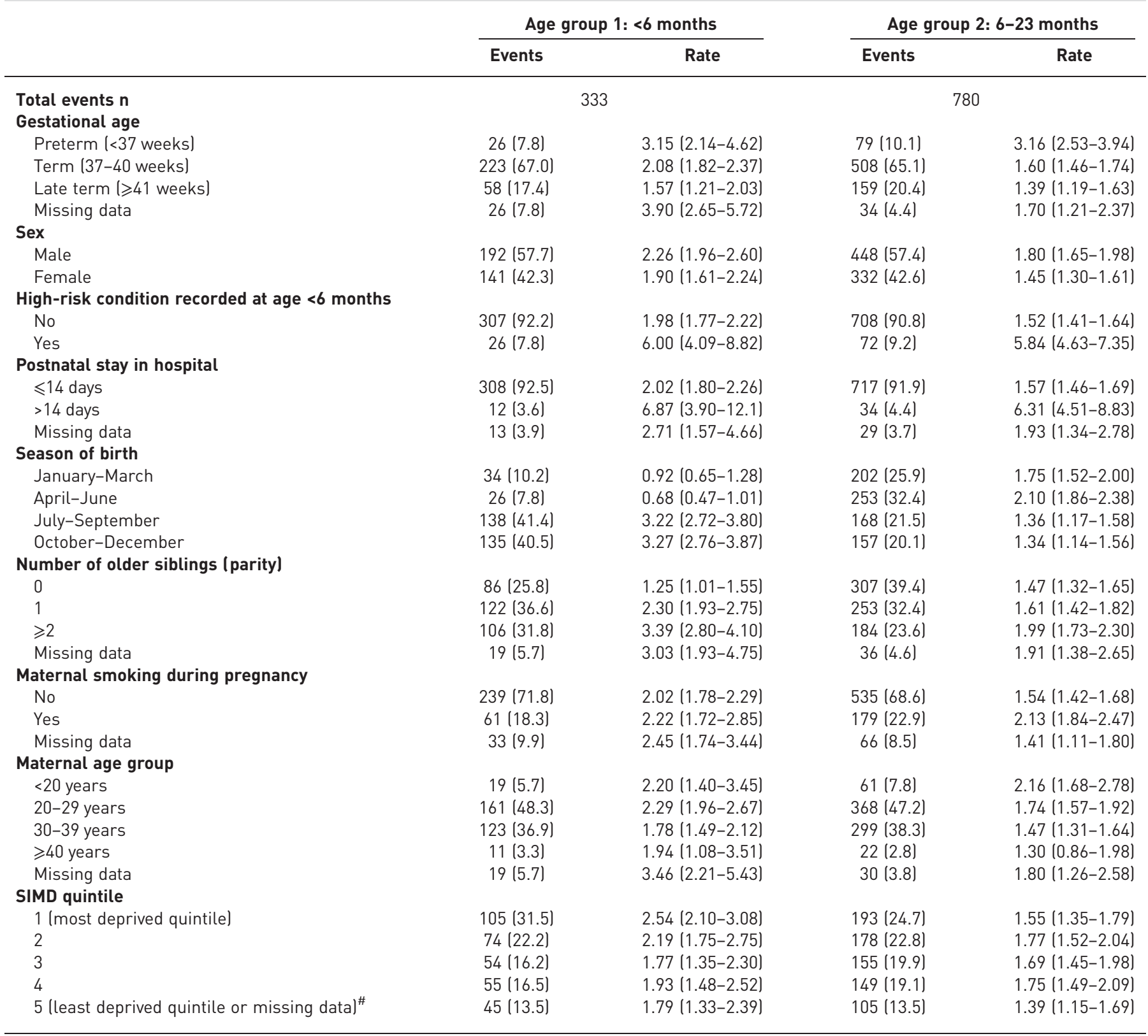

Data are presented as $\mathrm{n}(\%)$ or rate per 1000 child-years (95\% CI), unless otherwise stated. SIMD: Scottish Index of Multiple Deprivation.

\#: fewer than five children had missing information on SIMD quintile.

laboratory-confirmed influenza admission rates are higher than rates reported by studies relying only on clinical coding [38, 39]. We examined risk factors across six influenza seasons combined to ensure sufficient numbers of influenza-associated hospital admissions. Since our results were based on data from $\mathrm{A} / \mathrm{H} 1 \mathrm{~N} 1, \mathrm{~A} / \mathrm{H} 3 \mathrm{~N} 2$ and B-dominant seasons, they should be broadly representative across multiple influenza seasons with a similar strain circulation pattern.

This is the first study to specifically examine multiple risk factors for influenza in children aged $<6$ months. We found that the presence of older siblings and season of birth were the strongest risk factors for influenza-confirmed admission in this age group, with PAFs of $46 \%$ and $66 \%$, respectively. Higher parity has previously been identified as a risk factor for respiratory infection-related hospital admissions [40]. Since there is no influenza vaccine licensed for this young age group, reducing the risk of influenza complications will rely on improving vaccination uptake among siblings and pregnant women. Pregnant 
TABLE 3 Crude and adjusted hazard ratios (HR) from Cox proportional hazards model (based on multiply-imputed datal for influenza-confirmed admissions in children aged $<6$ months lage group 1)

Crude HR $(95 \% \mathrm{CI})$

Adjusted HR $(95 \% \mathrm{CI})$

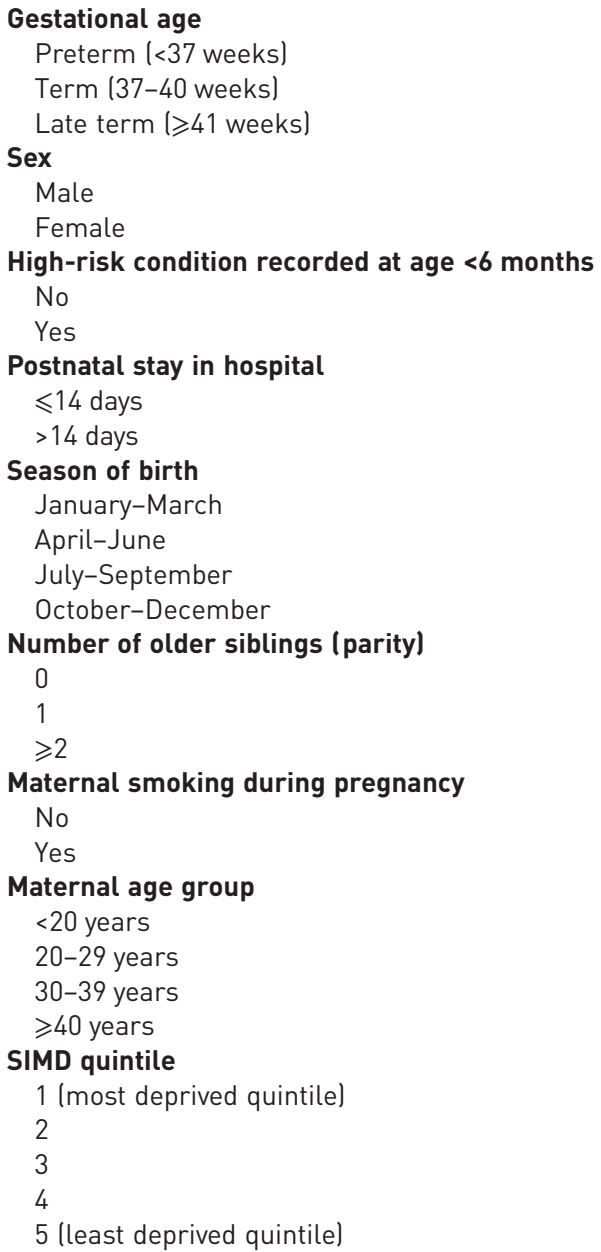

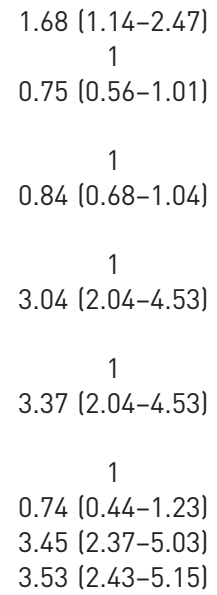

1

$1.82(1.38-2.40)$

$2.72(2.06-3.61)$

1

$1.12(0.85-1.49)$

$1.24(0.77-2.00)$

$1.28(1.01-1.61)$

1

$1.08(0.59-2.00)$

$1.48(1.04-2.11)$

$1.28(0.88-1.86)$

$1.03(0.69-1.53)$

$1.12(0.75-1.67)$
1.29 (0.82-2.04)

$0.84(0.63-1.12)$

$0.86(0.69-1.06)$

1

$2.46(1.59-3.80)$

1

$1.79(0.88-3.67)$

1

$0.74(0.44-1.23)$

$3.49(2.40-5.08)$

3.56 (2.44-5.19)

1

$2.02(1.52-2.69)$

$3.13(2.32-4.22)$

$0.86(0.64-1.15)$

1.98 (1.20-3.29)

1.47 (1.15-1.87)

$0.94(0.51-1.74)$

$1.28(0.89-1.84)$

$1.15(0.79-1.69)$

$0.97(0.65-1.45)$

$1.10(0.74-1.64)$

1

Events $n=333$; subjects $n=343068$. SIMD: Scottish Index of Multiple Deprivation.

women and parents of children aged $<2$ years should be encouraged to vaccinate older siblings if they are eligible under the universal programme. In addition, pregnant women should be given information about the influenza risk reduction in newborns conferred by maternal influenza vaccination. One clinical trial has indicated that the effects may only last for $<2$ months [8]. In our study, $26 \%$ of admissions in children aged $<6$ months occurred in the first 2 months of life. Thus, maternal influenza vaccine would not prevent the majority of admissions in this age group. Further research into the duration of maternal influenza vaccine protection in infants, and efforts to develop influenza vaccines for children aged $<6$ months are required.

Parity and season of birth were significantly associated with admission risk in children aged 6-23 months. However, the associations with influenza hospital admission risk were weaker, most likely because of greater mixing through childcare. None of the risk factors were associated with a PAF of $>20 \%$ in this age group, indicating that targeting specific risk groups (such as children with older siblings or children born prematurely) is unlikely to prevent the majority of cases. Instead, extending the universal influenza vaccination programme to children aged 6-23 months would prevent more hospital admissions in this age group. Vaccination of all children aged 6-23 months is not recommended in the UK, despite evidence of cost-effectiveness [41], due to the perceived lack of evidence of effectiveness of inactivated influenza vaccines in this age group. 
TABLE 4 Crude and adjusted hazard ratios (HR) from Cox proportional hazards model (based on multiply-imputed datal for influenza-confirmed admissions in children aged 6-23 months lage group 2)

Crude HR $(95 \% \mathrm{CI})$

Adjusted HR $(95 \% \mathrm{CI})$

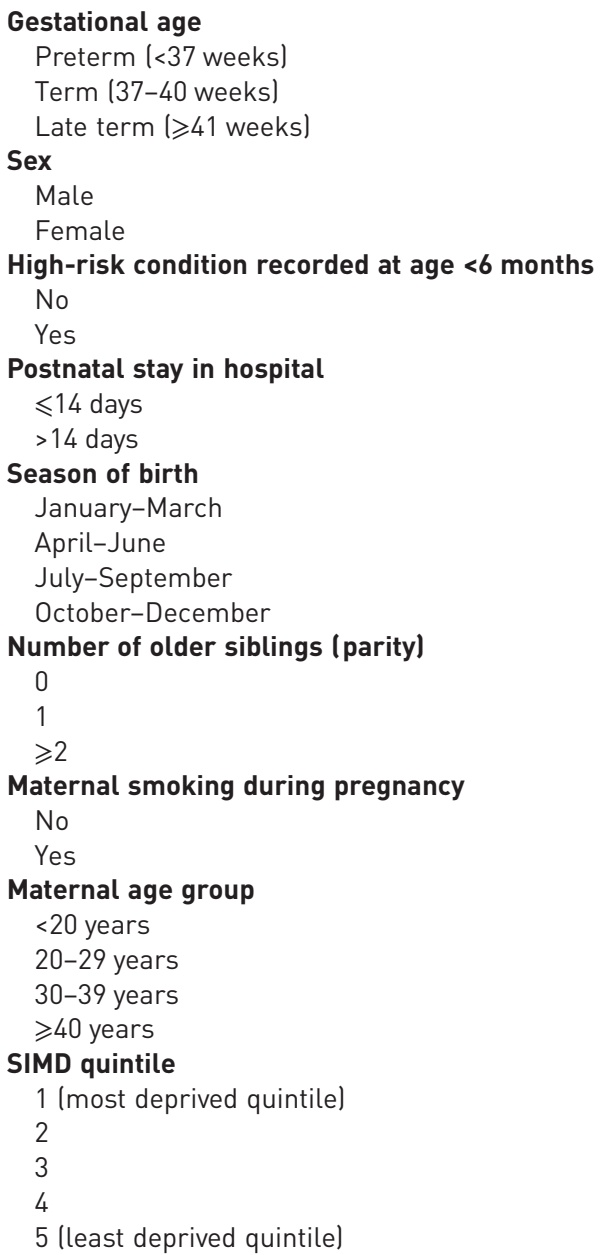

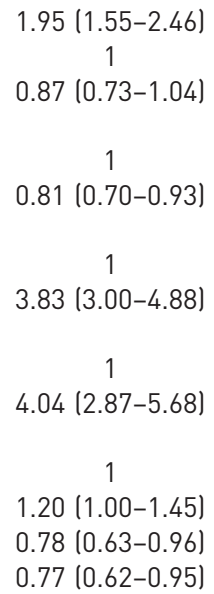

$1.09(0.93-1.29)$

$1.37(1.14-1.64)$

1

$1.39(1.17-1.66)$

$1.48(1.13-1.95)$

$1.18(1.01-1.38)$

1

0.89 (0.58-1.37)

$1.10(0.87-1.40)$

1.25 (0.99-1.60)

$1.20(0.94-1.54)$

1.16 (0.97-1.59)
$1.36(1.03-1.80)$

$0.90(0.75-1.07)$

$0.82(0.71-0.95)$

1

$3.04(2.33-3.96)$

1

$1.82(1.18-2.80)$

1

$1.21(1.00-1.45)$

0.78 (0.64-0.96)

0.77 (0.63-0.95)

1

$1.18(0.99-1.40)$

1.49 (1.23-1.80)

1

1.25 (1.03-1.50)

1.65 (1.23-2.23)

1.24 (1.05-1.46)

$0.81(0.53-1.25)$

0.89 (0.69-1.14)

$1.08(0.84-1.38)$

$1.09(0.85-1.41)$

$1.18(0.92-1.52)$

Events n=780; subjects n=399 454. SIMD: Scottish Index of Multiple Deprivation.

Since universal vaccination of school-aged children is predicted to reduce influenza transmission overall [41], the risk of influenza admission by season of birth and parity in children aged $<2$ years may attenuate over the coming years. These risks should be monitored as part of the evaluation of the universal paediatric influenza vaccine programme in the UK. As evidence of the population impact of universal influenza vaccine and effectiveness of inactivated influenza vaccines in young children emerges [18], there may be a need to reconsider the extension of influenza vaccination to children aged $<2$ years. However, this study only includes influenza-confirmed hospital admissions, and we do not examine risk factors for influenza infection that do not require healthcare intervention, or those presenting only in primary care. Furthermore, although our results indicate that a targeted vaccination programme may not reduce the majority of influenza-associated hospital admissions in children with high-risk conditions, children in clinical high-risk groups are over-represented among influenza-related intensive-care admissions and deaths $[42,43]$. Any reconsideration of the influenza vaccination programme for children aged 6-23 months will need to take into account the full range of severity of influenza-related illness in children as well as social impacts, including time off work for parents.

In agreement with a previous systematic review [24], we found that children born preterm were more likely to be admitted to hospital with influenza than children born at or after term. However, the increased risk was only significant among children aged 6-23 months. Children born prematurely without other 
TABLE 5 Population-attributable fractions of the proportion of admissions prevented by setting the named risk factors to the value of the specified categories

Risk factor and scenario"

Population-attributable fraction

$\begin{array}{lc}\text { Age group 1: }<6 \text { months } & \\ \text { Parity: first child } & 45.5(33.5-55.3) \\ \text { Maternal age: } 30-39 \text { years } & 18.5(5.5-29.7) \\ \text { High risk: no } & 4.5(1.3-7.7) \\ \text { Season of birth: April-June } & 65.8(49.9-76.6) \\ \text { Age group 2: 6-23 months } & \\ \text { Parity: first child } & 13.7(5.2-21.4) \\ \text { Maternal age: } 30-39 \text { years } & 11.4(2.5-21.4) \\ \text { High risk: no } & 5.6(3.5-7.7) \\ \text { Season of birth: October-December } & 19.2(6.4-30.3) \\ \text { Gestational age: } \geqslant 41 \text { weeks } & 9.9(-3.8-21.7) \\ \text { Maternal smoking during pregnancy: no } & 4.9(0.6-9.1) \\ \text { Postnatal stay in hospital: <14 days } & 1.5(0-3)\end{array}$

Data are presented as $\%(95 \% \mathrm{CI}) .{ }^{\#}$ : note that all other variables are held constant on their observed values in the data in the main adjusted models.

high-risk conditions are currently not recommended to receive influenza vaccine in the UK. This study adds to the growing evidence [24] to support an extension of the targeted vaccination programmes to children born prematurely and aged 6-23 months. Larger studies are required to examine the risk of admission according to more specific groups of gestational age.

Vaccination of children who are eligible for influenza vaccination under a universal programme (children aged $\geqslant 2$ years in the UK) who have a sibling aged $<2$ years during the influenza season should be strongly encouraged to be vaccinated in order to prevent influenza-associated hospital admissions among young children. Further research is required into the duration of maternal antibody protection and influenza vaccine development for children aged $<6$ months.

\section{Acknowledgements}

We gratefully acknowledge the help of Carole Morris at the Electronic Data Research and Innovation Service (eDRIS; Edinburgh, UK) in extracting and linking the data for this study, and the Electronic Communication of Surveillance in Scotland (ECOSS) team at Health Protection Scotland (Glasgow, UK) for supplying the laboratory surveillance data.

\section{References}

1 Nair H, Brooks WA, Katz M, et al. Global burden of respiratory infections due to seasonal influenza in young children: a systematic review and meta-analysis. Lancet 2011; 378: 1917-1930.

2 Neuzil KM, Mellen BG, Wright PF, et al. The effect of influenza on hospitalizations, outpatient visits, and courses of antibiotics in children. $N$ Engl J Med 2000; 342: 225-231.

3 Iwane MK, Edwards KM, Szilagyi PG, et al. Population-based surveillance for hospitalizations associated with respiratory syncytial virus, influenza virus, and parainfluenza viruses among young children. Pediatrics 2004; 113: $1758-1764$.

4 Bonati M, Clavenna A. Seasonal influenza immunization in early infancy? BMC Public Health 2012; 12: 873.

5 Madhi SA, Cutland CL, Kuwanda L, et al. Influenza vaccination of pregnant women and protection of their infants. N Engl J Med 2014; 371: 918-931.

6 Zaman K, Roy E, Arifeen SE, et al. Effectiveness of maternal influenza immunization in mothers and infants. $N$ Engl J Med 2008; 359: 1555-1564.

7 Shakib J, Korgenski K, Presson A, et al. Influenza in infants born to women vaccinated during pregnancy. Pediatrics 2016; 137: e20152360.

8 Nunes MC, Cutland CL, Jones S, et al. Duration of infant protection against influenza illness conferred by maternal immunization: secondary analysis of a randomized clinical trial. JAMA Pediatr 2016; 170: 840-847.

9 Grohskopf LS, Sokolow LZ, Broder KR, et al. Prevention and control of seasonal influenza with vaccines. MMWR 2016; 65: 1-54

10 National Advisory Committee on Immunization. Canadian Immunization Guide Chapter on Influenza and Statement on Seasonal Influenza Vaccine for 2016-2017. www.phac-aspc.gc.ca/naci-ccni/assets/pdf/ flu-2016-2017-grippe-eng.pdf Date last accessed: November 15, 2016. Date last updated: May 2015.

11 Government of Western Australia Department of Health. Disease Watch: Child Influenza Vaccination Rate Low Despite Rise in Uptake. www.health.wa.gov.au/diseasewatch/vol17_issue4/child_vaccination.cfm Date last accessed: October 01, 2015. Date last updated: December 2013.

12 Australian Government Department of Health. Immunise Australia Programme: Influenza. www.immunise.health gov.au/internet/immunise/publishing.nsf/Content/immunise-influenza Date last accessed: December 16, 2016. Date last accessed: March 12, 2017. 
13 European Centre for Disease Prevention and Control. Seasonal Influenza Vaccination and Antiviral Use in Europe. 2016 https:/ecdc.europa.eu/sites/portal/files/media/en/publications/Publications/Seasonal-influenzavaccination-antiviral-use-europe.pdf Date last accessed: July 24, 2017.

14 Immunisation Advisory Centre. Influenza vaccines - Eligibility Criteria. www.influenza.org.nz/eligibility-criteria Date last accessed: December 16, 2016.

15 Rajaram S, Steffey A, Blak B, et al. Uptake of childhood influenza vaccine from 2012-2013 to 2014-2015 in the UK and the implications for high-risk children: a retrospective observational cohort study. BMJ Open 2016; 6: $\mathrm{e} 010625$.

16 Moran K, Maaten S, Guttmann A, et al. Influenza vaccination rates in Ontario children: implications for universal childhood vaccination policy. Vaccine 2009; 27: 2350-2355.

17 Campitelli MA, Inoue M, Calzavara AJ, et al. Low rates of influenza immunization in young children under Ontario's universal influenza immunization program. Pediatrics 2012; 129: e1421-e1430.

18 Blyth CC, Jacoby P, Effler PV, et al. Effectiveness of trivalent flu vaccine in healthy young children. Pediatrics 2014; 133: e1218-e1225.

19 Centers for Disease Control and Prevention. Flu Vaccination Coverage: United States, 2014-15 Influenza Season. www.cdc.gov/flu/pdf/fluvaxview/nfid-coverage-2014-15-final.pdf Date last accessed: December 16, 2016. Date last updated: September 17, 2015.

20 Public Health England. Influenza Immunisation Programme for England: GP Patient Groups. Data Collection Survey Season 2015 to 2016. www.gov.uk/government/uploads/system/uploads/attachment_data/file/544552/ Seasonal_flu_GP_patient_groups_annual_report_2015_2016.pdf Date last accessed: October 17, 2016. Date last updated: May 2016.

21 Public Health England. Surveillance of Influenza and Other Respiratory Viruses in the United Kingdom: Winter 2015 to 2016. www.gov.uk/government/uploads/system/uploads/attachment_data/file/526405/Flu_Annual_Report_ 2015 2016.pdf Date last accessed: December 10, 2016. Date last updated: May 2016.

22 Regan AK, Mak DB, Hauck YL, et al. Trends in seasonal influenza vaccine uptake during pregnancy in Western Australia: implications for midwives. Women Birth 2016; 29: 423-429.

23 Centers for Disease Control and Prevention. Flu Vaccination Coverage Among Pregnant Women - United States, 2015-16 Flu Season. www.cdc.gov/flu/fluvaxview/pregnant-coverage_1516estimates.htm Date last accessed: January 26, 2017. Date last updated: September 29, 2016.

24 Gill PJ, Ashdown HF, Wang K, et al. Identification of children at risk of influenza-related complications in primary and ambulatory care: a systematic review and meta-analysis. Lancet Respir Med 2015; 3: 139-149.

25 Navaranjan D, Rosella LC, Kwong JC, et al. Ethnic disparities in acquiring 2009 pandemic H1N1 influenza: a case-control study. BMC Public Health 2014; 14: 214

26 Dharan NJ, Sokolow LZ, Cheng PY, et al. Child, household, and caregiver characteristics associated with hospitalization for influenza among children 6-59 months of age: an emerging infections program study. Pediatr Infect Dis J 2014; 33: e141-e150.

27 Launes C, García-García JJ, Martínez-Planas A, et al. 2009 H1N1: risk factors for hospitalization in a matched case-control study. Eur J Pediatr 2012; 171: 1127-1131.

28 Scottish Government. Scottish Index of Multiple Deprivation. www.gov.scot/Topics/Statistics/SIMD Date last accessed: January 05, 2017.

29 Cromer D, van Hoek AJ, Jit M, et al. The burden of influenza in England by age and clinical risk group: a statistical analysis to inform vaccine policy. J Infect 2014; 68: 363-371.

30 PRIMIS. Seasonal Influenza Vaccine Uptake Reporting Specification Collection 2014/2015. www.nottingham.ac.uk/ primis/documents/specs/seasonal-flu-lqd-specification-14-15-v7.pdf Date last accessed: October 01, 2015. Date last updated: July 31, 2014.

31 Health Protection Scotland. National Influenza Reports. 2017 www.hps.scot.nhs.uk/resp/influenzareports.aspx Date last accessed: February 09, 2017. Date last updated: June 27, 2017.

32 White I, Royston P. Imputing missing covariate values for the Cox model. Stat Med 2009; 28: 1982-1998

33 Newson R. Attributable and unattributable risks and fractions and other scenario comparisons. Stata J 2013; 13: $672-698$

34 Moore HC, Lehmann D, de Klerk N, et al. How accurate are International Classification of Diseases-10 diagnosis codes in detecting influenza and pertussis hospitalizations in children? J Pediatric Infect Dis Soc 2014; 3: 255-260.

35 Nicholson KG, McNally T, Silverman M, et al. Rates of hospitalisation for influenza, respiratory syncytial virus and human metapneumovirus among infants and young children. Vaccine 2006; 24: 102-108.

36 Haerskjold A, Kristensen K, Kamper-Jørgensen M, et al. risk factors for hospitalization for respiratory syncytial virus infection: a population-based cohort study of Danish children. Pediatr Infect Dis J 2016; 35: 61-65.

37 Grijalva CG, Craig AS, Dupont WD, et al. Estimating influenza hospitalizations among children. Emerging Infect Dis 2006; 12: 103-109.

38 Matias G, Taylor R, Haguinet F, et al. Modelling estimates of age-specific influenza-related hospitalisation and mortality in the United Kingdom. BMC Public Health 2016; 16: 481.

39 Beard F, McIntyre P, Gidding H, et al. Influenza related hospitalisations in Sydney, New South Wales, Australia. Arch Dis Child 2006; 91: 20-25.

40 Moore HC, de Klerk N, Richmond P, et al. A retrospective population-based cohort study identifying target areas for prevention of acute lower respiratory infections in children. BMC Public Health 2010; 10: 757.

41 Baguelin $\mathrm{M}$, Flasche S, Camacho A, et al. Assessing optimal target populations for influenza vaccination programmes: an evidence synthesis and modelling study. PLoS Med 2013; 10: e1001527.

42 Sachedina N, Donaldson LJ. Paediatric mortality related to pandemic influenza A H1N1 infection in England: an observational population-based study. Lancet 2010; 376: 1846-1852.

43 Dalziel SR, Thompson JM, Macias CG, et al. Predictors of severe H1N1 infection in children presenting within Pediatric Emergency Research Networks (PERN): retrospective case-control study. BMJ 2013; 347 : f4836. 